\title{
Querying ontology using keywords and quantitative restriction phrases
}

\begin{abstract}
Many approaches for converting keyword queries to formal query languages are presented for natural language interfaces to ontologies. Some approaches present fixed formal query templates, so they lack in providing support with increasing number of words in the user query. Other approaches work on constructing and manipulating subgraphs from RDF graphs so their processing is complex with respect to time and space. Techniques are presented to perform operations by obtaining a reduced RDF graph but they limit the input to some type of resources so their complete complexity with all type of input resources is unknown. For formal query generation, we present a variable query template whose computation is facilitated by less complex and distributed RDF property and relation graphs. A prototype QuriOnto is developed to evaluate our design. The user can query QuriOnto with any number of words and resource types. Also, to the best of our knowledge, it is the first system that can handle quantitative restrictions with keyword queries. As QuriOnto has no support for semantic similarity at this time except for rdfs labels so its recall is low but high precision shows that the approach is promising for the generation of corresponding formal queries.
\end{abstract}

Keyword: Natural language interfaces; Ontology; Keyword search; SPARQL; Query; Semantic web 